\title{
On Monomial Golod Ideals
}

\author{
Hailong Dao ${ }^{1} \cdot$ Alessandro De Stefani $^{2}$ (iD
}

Received: 20 May 2019 / Revised: 12 March 2020 / Accepted: 19 May 2020 /

Published online: 24 September 2020

(C) The Author(s) 2020

\begin{abstract}
We study ideal-theoretic conditions for a monomial ideal to be Golod. For ideals in a polynomial ring in three variables, our criteria give a complete characterization. Over such rings, we show that the product of two monomial ideals is Golod.
\end{abstract}

Keywords Golod rings · Product of ideals $\cdot$ Koszul homology $\cdot$ Koszul cycles

Mathematics Subject Classification (2010) Primary 13D02 · Secondary 05E40

\section{Introduction}

Let $k$ be a field, and let $Q=k\left[x_{1}, \ldots, x_{n}\right]$ be a polynomial ring on $n$ variables over $k$, with $\operatorname{deg}\left(x_{i}\right)=1$ for all $i$. We denote by $\mathfrak{m}=\left(x_{1}, \ldots, x_{n}\right)$ the homogeneous maximal ideal of $Q$. Let $I \subseteq \mathfrak{m}^{2}$ be a homogeneous ideal and $R=Q / I$. Serre proved a coefficient-wise inequality of formal power series for the Poincare series of $R$ :

$$
P_{k}^{R}(t):=\sum_{i \geq 0} \operatorname{dim}_{k} \operatorname{Tor}_{i}^{R}(k, k) t^{i} \ll \frac{(1+t)^{n}}{1-\sum_{i>0} \operatorname{dim}_{k} \operatorname{Tor}_{i}^{Q}(k, R) t^{i+1}} .
$$

When equality happens, the ring $R$ (and the ideal $I$ ) is called Golod. The notion is defined and studied extensively in the local setting, but in this paper we shall restrict ourselves to the graded situation. Golod rings and ideals have attracted increasing attention recently (see [6, $8,9,12,15]$ ), but they remain mysterious even when $n=3$. For instance, we do not know if the product of any two homogeneous ideals in $Q=k[x, y, z]$ is Golod. Another reason for the increasing interest is their connection to moment-angle complexes (for example, see $[7,10,14])$.

Alessandro De Stefani

destefani@dima.unige.it

Hailong Dao

hdao@ku.edu

1 Department of Mathematics, The University of Kansas, Lawrence, KS 66045, USA

2 Dipartimento di Matematica, Università di Genova, Via Dodecaneso 35, 16146 Genova, Italy 
It was asked by Welker whether it is always the case that the product of two proper homogeneous ideals is Golod (for example, see [16, Problem 6.18]) but a counter-example, even for monomial ideals, was constructed by the second author in [8].

In this work, we provide a concrete characterization of Golod monomial ideals in three variables, and use it to show that the product of any two proper monomial ideals in $Q=$ $k[x, y, z]$ is Golod. The following is our first main result:

Theorem 1.1 Let $Q=k[x, y, z]$ and $I \subseteq \mathfrak{m}^{2}$ be a monomial ideal. Then, $I$ is Golod if and only if the following conditions hold:

(1) $\left[I: x_{1}\right] \cdot\left[I:\left(x_{2}, x_{3}\right)\right] \subseteq I$ for all permutations $\left\{x_{1}, x_{2}, x_{3}\right\}$ of $\{x, y, z\}$.

(2) $\left[I: x_{1}\right] \cdot\left[I: x_{2}\right] \subseteq x_{3}\left[I:\left(x_{1}, x_{2}\right)\right]+I$ for all permutations $\left\{x_{1}, x_{2}, x_{3}\right\}$ of $\{x, y, z\}$.

We point out that rings with embedding codepth at most three have been studied extensively. For instance, their Koszul homology has been completely classified up to isomorphism (see [1, 3-5, 18]).

To obtain Theorem 1.1, we first list in Proposition 2.1 a set of necessary conditions for Golodness for general ideals in any number of variables, that are easy to check and are of independent interest. They can be used to provide quick examples of non-Golod ideals. For the sufficiency of the conditions in Theorem 1.1, the fact that $I$ is a monomial ideal is crucial. In fact, there are examples of homogeneous ideals in $k[x, y, z]$ that satisfy all the conditions of the theorem, but are not Golod (see Example 2.7).

Our second main result is a consequence of Theorem 1.1: we obtain that products of monomial ideals in three variables are Golod.

Corollary 1.2 Let $J, K$ be proper monomial ideals in $Q=k[x, y, z]$. Then, $I=J K$ is Golod.

Observe that this result is optimal, as the example of a non-Golod product of two monomial ideals constructed in [8] is in four variables. We end the paper with some positive and negative partial results regarding the colon conditions highlighted by this work, and several open questions.

\section{Characterization of Monomial Golod Ideals in Three Variables}

In this section, we prove Theorem 1.1. We first focus on the necessary part, which holds quite generally. Let $Q=k\left[x_{1}, \ldots, x_{n}\right], I$ a homogeneous ideal in $Q$, and $R=Q / I$. Let $K^{Q}$ be the Koszul complex on a minimal set of generators $x_{1}, \ldots, x_{n}$ of the maximal ideal $\mathfrak{m}$ of $Q$, and $K^{R}=R \otimes_{Q} K^{Q}$. The Koszul complex can be realized as the exterior algebra $\bigwedge K_{1}^{R}$, where $K_{1}^{R}$ is a free $R$-module of rank $n$, with basis $e_{x_{1}}, \ldots, e_{x_{n}}$. An element of the $p$-th graded component $K_{p}^{R}$ can be written as a sum of elements of the form $r e_{x_{i_{1}} \ldots x_{i_{p}}}$, where $1 \leq i_{1}<i_{1}<\cdots<i_{p} \leq n, r \in R$, and where we set $e_{x_{i_{1}} \ldots x_{i_{p}}}:=$ $e_{x_{i_{1}}} \wedge \ldots \wedge e_{x_{i_{p}}}$. The Koszul complex also comes equipped with a differential $\partial$, as it is a DG algebra. The differential is such that $\partial\left(e_{x_{i_{1}} \ldots x_{i_{p}}}\right)=\sum_{j=1}^{p}(-1)^{j-1} x_{i_{j}} e_{x_{i_{1}} \ldots \widehat{x_{i_{j}}} \ldots x_{i_{p}}}$, and extended by linearity to $K_{p}^{R}$. It is well-known that, if $I$ is Golod, then the product on the Koszul homology $H_{\geq 1}\left(K^{R}\right)$ is trivial (for example, see [2, Remark 5.2.1]). In other words, $R$ is Golod only if the map $H_{j}\left(K^{R}\right) \times H_{i-j}\left(K^{R}\right) \rightarrow H_{i}\left(K^{R}\right)$ is zero for all $1 \leq j \leq i \leq n$. 
Proposition 2.1 Let $Q=k\left[x_{1}, \ldots, x_{n}\right]$, and $I \subseteq \mathfrak{m}^{2}$ be a homogeneous ideal such that $R=Q / I$ is Golod. Then, the following hold:

(1) For any $1 \leq p \leq n$, we have

$$
\left[I:\left(x_{1}, \ldots, x_{p}\right)\right]\left[I:\left(x_{p+1}, \ldots, x_{n}\right)\right] \subseteq I .
$$

(2) For any $1 \leq p \leq n-1$, we have

$$
\left[I:\left(x_{1}, \ldots, x_{p}\right)\right]\left[I:\left(x_{p+1}, \ldots, x_{n-1}\right)\right] \subseteq x_{n}\left[I:\left(x_{1}, \ldots, x_{n-1}\right)\right]+I .
$$

Proof For (1), let $f \in I:\left(x_{1}, \ldots, x_{p}\right)$ and $g \in I:\left(x_{p+1}, \ldots, x_{n}\right)$. Then, by definition, the element $f e_{x_{1} \ldots x_{p}}$ is a cycle in $K_{p}^{R}$. Similarly for $g e_{x_{p+1} \ldots x_{n}}$. The product of these cycles is 0 in $H_{n}(R)$ if and only if $f g=0$ in $R$, which precisely says that $f g \in I$.

Similarly, take $f \in I:\left(x_{1}, \ldots, x_{p}\right)$ and $g \in I:\left(x_{p+1}, \ldots, x_{n-1}\right)$. Consider the cycles $f e_{x_{1} \ldots x_{p}}$ and $g e_{x_{p+1} \ldots x_{n-1}}$. The product of these is zero in $H_{n-1}(R)$ if and only if there is $h \in R$ such that $\partial\left(h e_{x_{1} \ldots x_{n}}\right)=f g e_{x_{1} \ldots x_{n-1}}$. But this means that $h x_{i}=0$ for $1 \leq i<n$ and $h x_{n}=f g$ in $R$. Lifting to $Q$, this shows that $h \in I:\left(x_{1}, \ldots, x_{n-1}\right)$, and thus $f g \in x_{n}[I$ : $\left.\left(x_{1}, \ldots, x_{n-1}\right)\right]+I$.

Remark 2.2 The above proposition is motivated by the examples in [8]. It can be used to easily provide examples of non-Golod ideals. For example, let $I=\left(x^{2}, y^{2}, z^{2}, t^{2}\right)(x, y, z, t) \subseteq$ $Q=k[x, y, z, t]$. Then, $x y \in I:(x, y)$ and $z t \in I:(z, t)$ but $x y z t \notin I$. Thus, $I$ is not Golod.

It is well-known that, for homogeneous ideals inside polynomial rings in three variables, being Golod is equivalent to requiring that the product on the Koszul homology is trivial (for instance, see [15, Theorem 6.3]). In the same article, it is shown that this is not the case more generally, even for monomial ideals. In order to prove the converse of Proposition 2.1 for monomial ideals in $k[x, y, z]$, we show that the Koszul homology modules admit "monomial bases". This is what we shall focus on for the rest of this section.

Definition 2.3 Let $Q=k\left[x_{1}, \ldots, x_{n}\right]$, and $I \subseteq \mathfrak{m}^{2}$ be a monomial ideal. Let $R=Q / I$. We say that $H_{p}\left(K^{R}\right)$ admits a monomial basis if it has a $k$-basis consisting of classes of cycles of the form $\bar{u} e_{x_{i_{1}} \ldots x_{i_{p}}}$, where $u \in Q$ is a monomial and $\bar{u}$ denotes its image inside $R$.

Clearly, if $\bar{u} e_{x_{i_{1}} \ldots x_{i_{p}}}$ is part of a monomial basis of $H_{p}\left(K^{R}\right)$, then $u \in I:\left(x_{i_{1}}, \ldots, x_{i_{p}}\right)$.

Observe that, if the ideal $I$ is homogeneous, then we can talk about homogeneous elements in $K^{R}$ : if $r \in R$ is homogeneous of degree $d$, then $\operatorname{deg}\left(r e_{x_{i_{1}} \ldots e_{x_{i}}}\right)=d+p$. In this case, the differential preserves degrees. Even more specifically, if $I$ is monomial, then each $K_{p}^{R}$ is a $\mathbb{Z}^{n}$-graded $R$-module. If $r=x_{1}^{a_{1}} \cdots x_{n}^{a_{n}}$, then $r e_{x_{i_{1}} \ldots x_{i_{p}}}$ has degree $\left(a_{1}, \ldots, a_{n}\right)+\epsilon_{i_{1}}+\cdots+\epsilon_{i_{p}}$, where $\epsilon_{j}$ is the vector in $\mathbb{Z}^{n}$ which has 1 in position $j$ and 0 elsewhere. For example, $x^{2} y^{3} e_{y z} \in K_{2}^{k[x, y, z]}$ has degree $(2,4,1)$. In this case, the differential $\partial$ on $K^{R}$ preserves multidegrees.

The following is well-known. Nonetheless, we provide a short proof for completeness.

Proposition 2.4 Let $Q=k\left[x_{1}, \ldots, x_{n}\right]$ and $I \subseteq \mathfrak{m}^{2}$ be a monomial ideal. Let $R=Q / I$. The modules $H_{\bullet}\left(K^{R}\right)$ admit a $\mathbb{Z}^{n}$-graded $k$-basis. 
Proof Since $I$ is a monomial ideal, $R$ admits a graded free resolution with $\mathbb{Z}^{n}$-graded shifts (for example, the Taylor resolution). There is a $\mathbb{Z}^{n}$-graded isomorphism

$$
H_{p}\left(K^{R}\right) \cong \operatorname{Tor}_{p}^{Q}(Q / I, k)
$$

that comes from tracing Koszul cycles along the double complex $P_{\bullet} \otimes K^{Q}$, where $P_{\bullet} \rightarrow R$ is a $\mathbb{Z}^{n}$-graded free resolution of $R$, and $K^{Q}$ can be viewed as a $\mathbb{Z}^{n}$-graded minimal free resolution of $Q / \mathfrak{m} \cong k$. Since $Q / I$ has $\mathbb{Z}^{n}$-graded shifts, we see that $\operatorname{Tor}_{p}^{Q}(Q / I, k)$ has a $\mathbb{Z}^{n}$-graded $k$-basis. Via this isomorphism, such a basis maps to a set of graded Koszul cycles in $K_{p}^{R}$, which forms a $k$-basis in homology.

We observe that if $\sum_{\left\{i_{1}, \ldots, i_{p}\right\} \subseteq[n]} r_{i_{1} \ldots i_{p}} e_{x_{i_{1}} \ldots x_{i_{p}}} \in K_{p}^{R}$ is $\mathbb{Z}^{n}$-graded, then each $r_{i_{1} \ldots i_{p}}$ must necessarily be a monomial. Furthermore, we must have $r_{i_{1} \ldots i_{p}} x_{i_{1}} \cdots x_{i_{p}}=$ $r_{i_{1}^{\prime} \ldots i_{p}^{\prime}} x_{i_{1}^{\prime}} \ldots x_{i_{p}^{\prime}}$ for all $\left\{i_{1}, \ldots, i_{p}\right\},\left\{i_{1}^{\prime}, \ldots, i_{p}^{\prime}\right\}$ for which $r_{i_{1} \ldots i_{p}} \neq 0$ and $r_{i_{1}^{\prime} \ldots i_{p}^{\prime}} \neq 0$. For example, $x e_{y z}+y e_{x z} \in K_{2}^{k[x, y, z]}$ is $\mathbb{Z}^{3}$-graded, of degree $(1,1,1)$.

Lemma 2.5 Let $Q=k\left[x_{1}, \ldots, x_{n}\right]$, and $I \subseteq \mathfrak{m}^{2}$ be a non-zero monomial ideal. Let $R=$ $Q / I$. There exists a $k$-basis of $H_{1}\left(K^{R}\right)$ consisting of elements of the form $\left\{u e_{x_{i}}\right\}$, where $u \in I: x_{i}$ is a monomial. Moreover, if $\operatorname{depth}(R)=0$, then there exists a $k$-basis of $H_{n}\left(K^{R}\right)$ consisting of elements of the form $\left\{\bar{u} e_{x_{1} \ldots x_{n}}\right\}$, where $u \in I: \mathfrak{m}$ is a monomial in $Q$ and $\bar{u}$ denotes its residue class in $R$.

Proof It is clear that a $k$-basis of $H_{n}\left(K^{R}\right)$ can be chosen to be of such form. In fact, an element of $K_{n}^{R}$ is of the form $\bar{f} e_{x_{1} \ldots x_{n}}$, where $f \in I: \mathfrak{m}$. Since $I$ is monomial, we can choose $f$ to be a monomial.

It is also fairly easy to prove the claim for $H_{1}\left(K^{R}\right)$ directly. However, we explain the process via lifting Koszul cycles, as this technique will be used later. Let $\left(P_{\bullet}, \delta\right)$ be a graded free resolution of $R$ as a $Q$-module. As noted in Proposition 2.4, we have a graded isomorphism between $\operatorname{Tor}_{1}^{Q}(Q / I, k)$ and $H_{1}\left(K^{R}\right)$. Let $I=\left(m_{1}, \ldots, m_{t}\right)$ be a minimal monomial generating set for $I$, and say that $m_{j}=x_{1}^{a_{1 j}} \cdots x_{n}^{a_{n j}}=: \underline{x}^{\underline{a}}$. Then, $\operatorname{Tor}_{1}^{Q}(Q / I, k) \cong \oplus_{j} k\left(-\underline{a}_{j}\right)$. The way the isomorphism goes is as follows

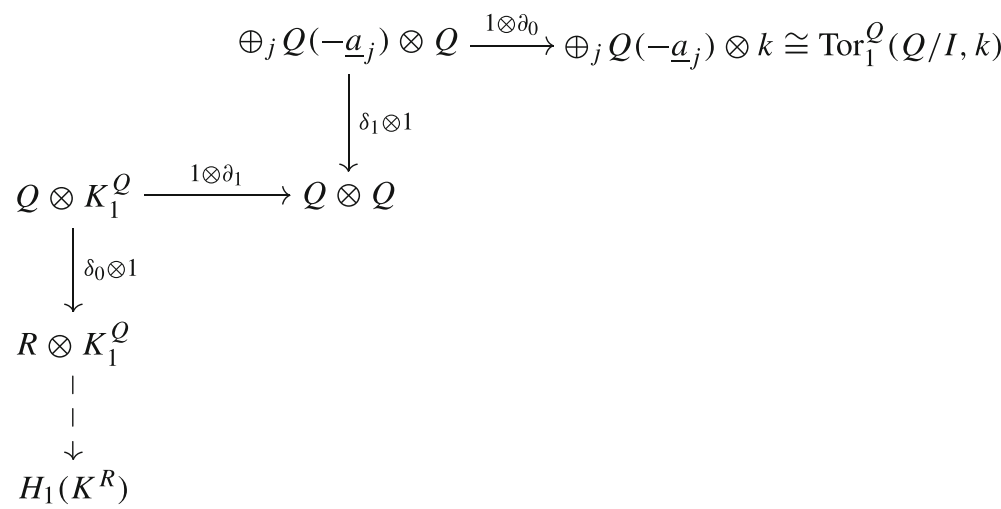

More explicitly, if we take a $\mathbb{Z}^{n}$-graded basis element of $k\left(-\underline{a}_{j}\right) \subseteq \operatorname{Tor}_{1}^{Q}(Q / I, k)$ and lift it to a basis element of $Q\left(-\underline{a}_{j}\right) \otimes Q$, then this will map down to $m_{j} \otimes 1 \in Q \otimes Q$ under $\delta_{1} \otimes 1$. If $x_{j}$ is any variable that divides $m_{j}$, say $m_{j}=x_{j} m_{j}^{\prime}$, then $m_{j} \otimes 1=(1 \otimes \partial)\left(m_{j}^{\prime} \otimes e_{x_{j}}\right)$, where $m_{j}^{\prime} \otimes e_{x_{j}} \in Q \otimes K_{1}^{Q}$. Applying $\delta_{0} \otimes 1$, we get the element $\overline{m_{j}^{\prime}} e_{x_{j}} \in K_{1}^{R} \otimes R \otimes Q$ which 
is a Koszul cycle. The process ends by considering its residue class in $H_{1}\left(K^{R}\right)$, which is a $k$-basis element of the desired form. Namely, a $k$-basis element of the form $\overline{m_{j}^{\prime}} e_{x_{j}}$ where $m_{j}^{\prime} \in I: x_{j}$ is a monomial.

Proposition 2.6 Let $I \subseteq Q=k[x, y, z]$ be a non-zero monomial ideal, and let $R=Q / I$. Then, for all $1 \leq p \leq 3-\operatorname{depth}(R)$, the module $H_{p}\left(K^{R}\right)$ admits a $k$-basis of the form $\left\{\bar{u} e_{x_{i_{1} \ldots x_{i p}}}\right\}$, where $u \in I:\left(x_{i_{1}}, \ldots, x_{i_{p}}\right)$ is a monomial, and $\bar{u}$ denotes the residue class of $u$ in $R$.

Proof The statement for $H_{1}\left(K^{R}\right)$ and $H_{3}\left(K^{R}\right)$ has already been proved in Lemma 2.5 (assuming that $\operatorname{depth}(R)=0$ for the latter to be non-zero). The argument for $H_{2}\left(K^{R}\right)$ exploits again the process of lifting Koszul cycles. Assume that $H_{2}\left(K^{R}\right) \neq 0$, that is, $\operatorname{depth}(R) \leq 1$. We consider a minimal $\mathbb{Z}^{3}$-graded free resolution of $R$ over $Q$

$$
F_{\bullet}: \ldots \longrightarrow \oplus_{\ell} Q\left(-\underline{b}_{\ell}\right) \stackrel{\delta_{2}}{\longrightarrow} \oplus_{j} Q\left(-\underline{a}_{j}\right) \stackrel{\delta_{1}}{\longrightarrow} Q \stackrel{\delta_{0}}{\longrightarrow} R \longrightarrow 0 .
$$

After fixing bases, $\delta_{1}$ can be represented as the matrix $\left[m_{1}, \ldots, m_{t}\right]$, where $\left\{m_{1}, \ldots, m_{t}\right\}$ is a minimal monomial generating set of $I$. On the other hand, $\delta_{2}$ is represented by a matrix where every column has precisely two non-zero monomial entries. This is because every relation between distinct monomials $m_{i}$ and $m_{j}$ is of this form $m_{i} u_{i}-m_{j} u_{j}=0$ for some monomials $u_{i}, u_{j} \in \mathfrak{m}$. We now describe the lifting process for $H_{2}\left(K^{R}\right)$. Consider the following part of double complex $F_{\bullet} \otimes K_{\bullet}^{Q}$ :

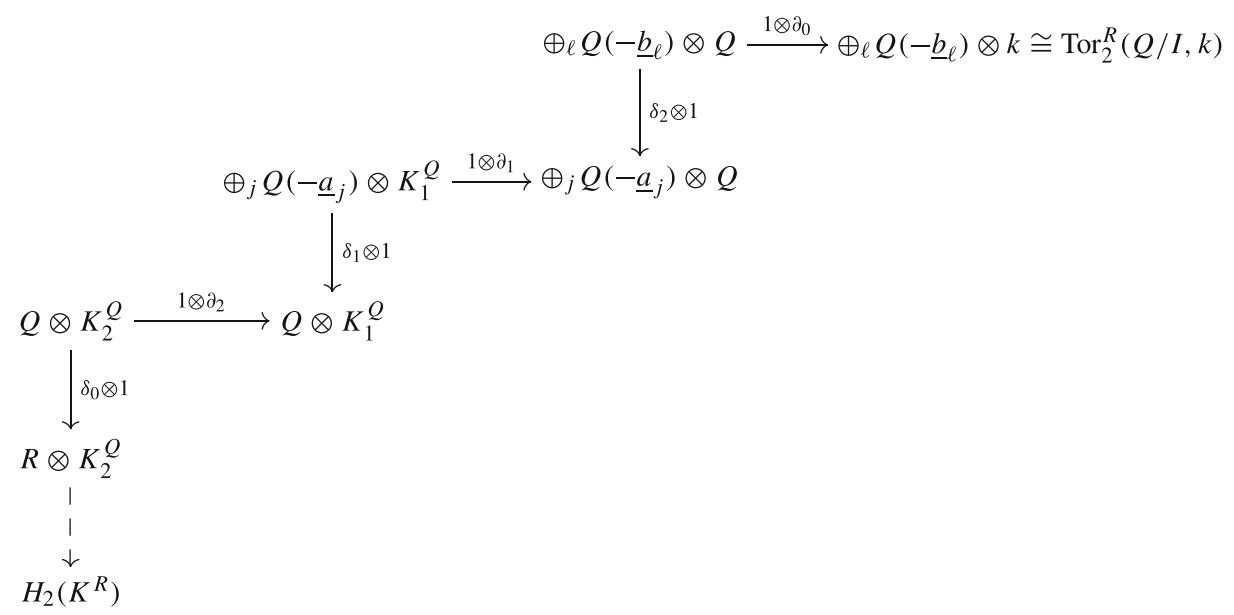

If we lift a $\mathbb{Z}^{3}$-graded basis element of $k\left(-\underline{b}_{\ell}\right) \subseteq \operatorname{Tor}_{2}^{R}(Q / I, k)$ to $\oplus_{\ell} Q\left(-\underline{b}_{\ell}\right) \otimes Q$, this will map down via $\delta_{2} \otimes 1$ to an element $\left(0, \ldots, u_{i}, \ldots,-u_{j}, \ldots, 0\right) \in \oplus_{j} Q\left(-\underline{a}_{j}\right) \cong$ $\bigoplus_{j} Q\left(-\underline{a}_{j}\right) \otimes Q$, corresponding to a binomial relation in the $\ell$-th column of the matrix representing $\delta_{2}$, as described above. Write $u_{i}=x_{i} v_{i}$ and $u_{j}=x_{j} v_{j}$, for some $i, j$, and monomials $v_{i}, v_{j} \in Q$. Observe that $i \neq j$, since otherwise the relation between $m_{i}$ and $m_{j}$ given by $\left(0, \ldots, u_{i}, \ldots,-u_{j}, \ldots, 0\right)$ would not be minimal. We may assume that $i<j$. Using the above relations, we have that $\left(0, \ldots, u_{i}, \ldots,-u_{j}, \ldots, 0\right)=(1 \otimes$ $\left.\partial_{1}\right)\left(0, \ldots, v_{i} e_{x_{i}}, \ldots,-v_{j} e_{x_{j}}, \ldots, 0\right)$. We now push this element down via $\delta_{1} \otimes 1$, to get an element $\sigma=v_{i} m_{i} \otimes e_{x_{i}}-v_{j} m_{j} \otimes e_{x_{j}} \in Q \otimes K_{1}^{Q}$. From $x_{i} v_{i} m_{i}=x_{j} v_{j} m_{j}$, we deduce that $v_{i} m_{i}=-x_{j} w$ for some monomial $w$. Consider the element $w \otimes e_{x_{i} x_{j}} \in Q \otimes K_{2}^{Q}$; 
we claim that $\left(1 \otimes \partial_{2}\right)\left(w \otimes e_{x_{i} x_{j}}\right)=\sigma$. By the definition of the differential, we have $\left(1 \otimes \partial_{2}\right)\left(w \otimes e_{x_{i} x_{j}}\right)=w x_{i} e_{x_{j}}-w x_{j} e_{x_{i}}$. Since $-w x_{j} x_{i}=v_{i} m_{i} x_{i}=v_{j} m_{j} x_{j}$, we deduce that $w x_{i}=-v_{j} m_{j}$. Putting these facts together gives $\left(1 \otimes \partial_{2}\right)\left(w \otimes e_{x_{i} x_{j}}\right)=$ $v_{i} m_{i} \otimes e_{x_{i}}-v_{j} m_{j} \otimes e_{x_{j}}=\sigma$, as desired. As $\left(\delta_{0} \otimes 1\right)\left(w \otimes e_{x_{i} x_{j}}=\bar{w} \otimes e_{x_{i} x_{j}}\right.$ is a cycle in $R \otimes K_{2}^{Q}$, the process of lifting Koszul cycles now ends by considering the class of the element $\bar{w} e_{x_{i} x_{j}}$ inside $H_{2}\left(K^{R}\right)$. As observed above, inside $Q$ we have $w x_{i}=-v_{j} m_{j} \in I$, and $w x_{j}=-v_{i} m_{i} \in I$. Therefore $w \in I:\left(x_{i}, x_{j}\right)$, and the class of $\bar{w} e_{x_{i} x_{j}}$ inside $H_{2}\left(K^{R}\right)$ gives then a basis element of the desired form.

Proof of Theorem 1.1 The necessary part was Proposition 2.1. But as Proposition 2.6 shows that all Koszul homologies admit a $k$-basis of the form $\left\{u e_{x_{i_{1} \ldots x_{p}}}\right\}$, where $u \in I$ : $\left(x_{i_{1}}, \ldots, x_{i_{p}}\right)$ is a monomial and $p=1,2,3$, the stated conditions are also sufficient.

There are examples of homogeneous ideals in $k[x, y, z]$ that satisfy the conditions of Theorem 1.1, but are not Golod.

Example 2.7 Let $Q=k[x, y, z]$, and let $\mathfrak{g}_{1}=\left(x y^{2}, x y z, y z^{2}, x^{4}-y^{3} z, x z^{3}-y^{4}\right)$. By [5, Theorem I] the ring $R=Q / \mathfrak{g}_{1}$ belongs to the class $G(2)$ in the classification of the Koszul homology of rings with embedding codepth at most three. In particular, $R$ is not Golod, by [3, 1.4.3]. However, it can be checked using Macaulay 2 [11] that the ideal $\mathfrak{g}_{1}$ satisfies conditions (1) and (2) of Theorem 1.1.

We observe that the condition that $I \subseteq \mathfrak{m}^{2}$ in Theorem 1.1 cannot be removed.

Example 2.8 Consider the ideal $I=\left(x, y^{2}, y z, z^{2}\right)$ inside $Q=k[x, y, z]$. This ideal does not satisfy the second condition of Theorem 1.1, since $y \in[I: x] \cdot[I: y]=I: y$, but $y \notin z[I:(x, y)]+I=I$. However, the ring $Q / I \cong k[y, z] /(y, z)^{2}$ is Golod.

Remark 2.9 Let $I$ be a monomial ideal in $k\left[x_{1}, \ldots, x_{n}\right]$. The condition "strongly Golod" considered by Herzog and Huneke in [12] means, in this context, that $\left[I:\left(x_{i}\right)\right]\left[I:\left(x_{j}\right)\right] \subseteq$ $I$ for all $1 \leq i, j \leq n$. This condition is clearly stronger than all the necessary colon conditions in Proposition 2.1. This makes sense, since strongly Golod implies Golod.

We conclude this section observing that, in the case of monomial ideals in four or more variables, some Koszul homology modules may not always admit a "monomial basis."

Example 2.10 Consider the ideal $I=(x z, x w, y z, y w)$ in $Q=k[x, y, z, w]$, and let $R=Q / I$. It is easy to check that $\alpha=x e_{y z w}-y e_{x z w}$ is a cycle of $K_{3}^{R}$, whose class equals that of $w e_{x y z}-z e_{x y w}$ in homology. Observe that $\alpha$ has multidegree $(1,1,1,1)$. Exploiting the multigrading, one can show that the class of $\alpha$ in homology cannot be expressed as a combination of elements coming from a monomial basis of $H_{3}\left(K^{R}\right)$.

\section{Products of Monomial Ideals in $k[x, y, z]$ are Golod}

In this section, we prove Corollary 1.2. By Theorem 1.1 and by the symmetry, it suffices to show the following lemmas. 
Lemma 3.1 Let $J, K$ be proper monomial ideals in $Q=k[x, y, z]$ and $I=J K$. Then

$$
[I: x][I:(y, z)] \subseteq I .
$$

Proof Let $f \in I: x$ and $g \in I:(y, z)$ be monomials. If $f \in(y, z)$, then $f g \in I$, so we assume $f=x^{a}$ for some $a \geq 0$. It follows that $x^{a+1} \in J K$, and as $J, K$ are proper we must have $f \in J \cap K$. As $J, K$ are monomial ideals, we have $g \in J K: y \subseteq(J: y) K+(K$ : y) $J \subseteq J+K$, and we are done.

Lemma 3.2 Let $J, K$ be proper monomial ideals in $Q=k[x, y, z]$ and $I=J K$. Then,

$$
(I: x)(I: y) \subseteq z[I:(x, y)]+I .
$$

Proof As $J$ and $K$ are monomial ideals, we have $J K: x=(J: x) K+(K: x) J$. We then have

$$
(J K: x)(J K: y)=J^{2}(K: x)(K: y)+K^{2}(J: x)(J: y)+J K(J: x)(K: y)+J K(K: x)(J: y) .
$$

By symmetry, it is enough to show that $J^{2}(K: x)(K: y) \subseteq z[J K:(x, y)]+J K$.

Let $J=J_{1}+z J^{\prime}$ with $J_{1}$ generated by $J \cap k[x, y]$. Then,

$$
J^{2}(K: x)(K: y) \subseteq J^{2}[K:(x, y)]=\left[J_{1}+z J^{\prime}\right] J[K:(x, y)] .
$$

But

$$
J_{1} J[K:(x, y)] \subseteq(x, y) J[K:(x, y)] \subseteq J K
$$

and

$$
z J^{\prime} J[K:(x, y)] \subseteq z J[K:(x, y)] \subseteq z[J K:(x, y)]
$$

\section{Integrally Closed Ideals and Some Questions}

The colon conditions considered in this paper seem related to the property of "being integrally closed" (see also the $\mathfrak{m}$-full and basically full conditions $[13,17]$ ). Here, we give some positive and negative results in this direction.

Lemma 4.1 Let $J, K$ be homogenous ideals in $Q=k[x, y, z]$ ( $J$ may not be proper) and $\mathfrak{m}=(x, y, z)$. Assume that $K \subseteq \mathfrak{m}^{2}$ and $K$ is integrally closed. Then if $I=J K$, we have $[I: x][I:(y, z)] \subseteq I$.

Proof First, observe that if $J K \subseteq(y, z)$, then $I: x=J K: x \subseteq(y, z): x=(y, z)$. From this, we deduce that $[I: x][I:(y, z)] \subseteq(y, z)[I:(y, z)] \subseteq I$, and the lemma holds in this case. Thus, we may assume that neither $J$ nor $K$ is contained in $(y, z)$. Write $(J, y, z)=\left(x^{a}, y, z\right)$ and $(K, y, z)=\left(x^{b}, y, z\right)$. Because of our assumptions, we have $a \geq 1$ and $b \geq 2$. Let $f \in J K: x$, so that $f x \in J K \subseteq(J, y, z)(K, y, z) \subseteq\left(x^{a+b}, y, z\right)$. It follows that $f \in\left(x^{a+b-1}, y, z\right)$, write $f=u x^{a+b-1}+v$ for some $v \in(y, z)$. Now let $g \in J K:(y, z)$, and consider the element $h=u x^{b-1} g$. As we are assuming $b \geq 2$, we have $h \in \mathfrak{m} J K:(y, z)$. On the other hand, since we have $f x=u x^{a+b}+x v \in J K$, we get that $x^{a+1} h=u x^{a+b} g=f x g-x v g \in \mathfrak{m} J K$. It follows that $h \in \mathfrak{m} J K:\left(x^{a+1}, y, z\right)$. Observe that $(\mathfrak{m} J, y, z)=\left(x^{a+1}, y, z\right)$. It follows that $h \in \mathfrak{m} J K:\left(x^{a+1}, y, z\right) \subseteq \mathfrak{m} J K: \mathfrak{m} J=K$, because $K$ is integrally closed. Since $f$ is congruent to $u x^{a+b-1}$ modulo $(y, z)$, we have 
that $f g$ is congruent to $u x^{a+b-1} g=x^{a} h$ modulo $J K$. On the other hand, $x^{a}$ belongs to $(J, y, z)$; therefore, $x^{a} h \in(J, y, z)[K \cap(J K:(y, z))] \subseteq J K$.

Corollary 4.2 Let I be a homogenous ideal in $Q=k[x, y, z]$ and $\mathfrak{m}=(x, y, z)$. Assume that $I \subseteq \mathfrak{m}^{2}$ and $I$ is integrally closed. Then, we have $[I: x][I:(y, z)] \subseteq I$.

Proof Let $J=R$ in Lemma 4.1.

Unfortunately, one can use other necessary colon criteria provided in Proposition 2.1 to show that even integrally closed monomial ideal in three variables or product of them in four variables may not be Golod.

Example 4.3 Let $I=\overline{\left(x^{2}, y^{4}, z^{4}, y z\right)}=\left(x^{2}, y^{4}, z^{4}, x z^{2}, y z, x y^{2}\right)$ in $Q=k[x, y, z]$. Then $x z \in(I: x)(I: y)$ but it is not in $z[I:(x, y)]+I$. So $I$ cannot be Golod by Proposition 2.1. If one does not want to restrict to $m$-primary ideals, a simpler example of an integrally closed ideal in $k[x, y, z]$ that is not Golod is $I=\left(x^{2}, y z\right)$. Indeed, this ideal fails again the condition $(I: x)(I: y) \subseteq z[I:(x, y)]+I$ of Proposition 2.1; moreover, it is a complete intersection of height two.

Example 4.4 Let $J=\overline{\left(x^{2}, y^{4}, z^{2}, y z\right)}$ and $K=\overline{\left(x^{4}, y^{2}, w^{2}, x w\right)}$ in $Q=k[x, y, z, w]$. Using Macaulay 2 [11], one can check that $J K$ is not Golod.

Note that both Examples 4.3 and 4.4 are in the smallest possible number of variables.

To end this paper, we pose some intriguing question motivated by our work. The obvious one is:

Question 4.5 Let $J, K$ be proper homogeneous ideals in $Q=k[x, y, z]$. Is $J K$ Golod?

We do not know the answer to Question 4.5 even when $K=\mathfrak{m}$. One can show that the conclusions of Lemmas 3.1 and 3.2 still hold when $K=\mathfrak{m}$ and $J$ is any proper homogeneous ideal in $Q$, so Proposition 2.1 does not provide any obstructions in this case. When the characteristic of $k$ is 0 and $J=K$, the answer is positive by the main result of [12].

Finally, we have not been able to determine whether Lemma 3.1 holds for any product $J K$ of homogeneous ideals in three variables, without either the monomial condition, or assuming that $K \subseteq \mathfrak{m}^{2}$ is integrally closed. Observe that any example for which the lemma fails would provide a negative answer to Question 4.5. It is rather frustrating that such a simple-looking question cannot be resolved, so the first author is willing to offer a cash prize of 25 USD for the first solver of this:

Question 4.6 Let $J, K$ be proper homogeneous ideals in $Q=k[x, y, z]$. Is this true that

$$
[I: x][I:(y, z)] \subseteq I
$$

for $I=J K$ ?

Acknowledgements We thank the anonymous referee for helpful comments on a previous version of this article. We thank Van Nguyen and Oana Veliche for many inspiring conversations about the content of this paper. We also thank Lucho Avramov for pointing out to us the classification of embedding codepth three rings. 
Funding Open access funding provided by Università degli Studi di Genova within the CRUI-CARE Agreement. The first author is partially supported by NSA grant H98230-16-1-001 during the preparation of this work.

Open Access This article is licensed under a Creative Commons Attribution 4.0 International License, which permits use, sharing, adaptation, distribution and reproduction in any medium or format, as long as you give appropriate credit to the original author(s) and the source, provide a link to the Creative Commons licence, and indicate if changes were made. The images or other third party material in this article are included in the article's Creative Commons licence, unless indicated otherwise in a credit line to the material. If material is not included in the article's Creative Commons licence and your intended use is not permitted by statutory regulation or exceeds the permitted use, you will need to obtain permission directly from the copyright holder. To view a copy of this licence, visit http://creativecommonshorg/licenses/by/4.0/.

\section{References}

1. Avramov, L.L., Kustin, A.R., Miller, M.: Poincaré series of modules over local rings of small embedding codepth or small linking number. J. Algebra 118(1), 162-204 (1988)

2. Avramov, L.L.: Infinite Free Resolutions. In: Six Lectures on Commutative Algebra (Bellaterra, 1996). Progr. Math.166, pp 1-118, Birkhäuser, Basel (1998)

3. Avramov, L.L.: A cohomological study of local rings of embedding codepth 3. J. Pure Appl. Algebra 216(11), 2489-2506 (2012)

4. Christensen, L.W., Veliche, O.: Local rings of embedding codepth 3: a classification algorithm. J. Softw. Algebra Geom 6, 1-8 (2014)

5. Christensen, L.W., Veliche, O.: Local rings of embedding codepth 3. Examples. Algebr. Represent. Theory 17(1), 121-135 (2014)

6. Christensen, L.W., Veliche, O.: The Golod property of powers of the maximal ideal of a local ring. Arch. Math. (Basel) 110(6), 549-562 (2018)

7. Denham, G., Suciu, A.I.: Moment-angle complexes, monomial ideals and Massey products. Pure Appl Math. Q.3,(1, Special Issue: In honor of Robert D. MacPherson. Part 3), 25-60 (2007)

8. De Stefani, A.: Products of ideals may not be Golod. J. Pure Appl. Algebra 220(6), 2289-2306 (2016)

9. Frankhuizen, R.: $A_{\infty}$-resolutions and the Golod property for monomial rings. Algebr. Geom. Topol. 18(6), 3403-3424 (2018)

10. Grbić, J., Panov, T., Theriault, S., Wu, J.: The homotopy types of moment-angle complexes for flag complexes. Trans. Am. Math. Soc. 368(9), 6663-6682 (2016)

11. Grayson, D.R., Stillman, M.E.: Macaulay2, a software system for research in algebraic geometry. Available at http://www.math.uiuc.edu/Macaulay2/

12. Herzog, J., Huneke, C.: Ordinary and symbolic powers are Golod. Adv. Math. 246, 89-99 (2013)

13. Heinzer, W.J., Ratliff Jr. L.J., Rush, D.E.: Basically full ideals in local rings. J. Algebra 250(1), 371-396 (2002)

14. Iriye, K., Kishimoto, D.: Golodness and polyhedral products of simplicial complexes with minimal Taylor resolutions. Homology Homotopy Appl. 20(1), 69-78 (2018)

15. Katthän, L.: A non-Golod ring with a trivial product on its Koszul homology. J. Algebra 479, 244-262 (2017)

16. McCullough, J., Peeva, I.: Infinite graded free resolutions. In: Commutative Algebra and Noncommutative Algebraic Geometry. Vol. I. Math. Sci. Res. Inst. Publ.67, 215-257. Cambridge Univ. Press, New York (2015)

17. Watanabe, J.: m-full ideals. Nagoya Math. J. 106, 101-111 (1987)

18. Weyman, J.: On the structure of free resolutions of length 3. J. Algebra 126(1), 1-33 (1989)

Publisher's Note Springer Nature remains neutral with regard to jurisdictional claims in published maps and institutional affiliations. 